\title{
Dépérissement du pin maritime en Vendée. Résistance au chlorure de sodium de 3 provenances géographiques dans différentes conditions édaphiques
}

\author{
E Saur, N Rotival, C Lambrot, P Trichet
}

INRA, Station de recherches forestières de Bordeaux-Cestas, BP 45, 33611 Gazinet Cedex, France

(Reçu le 30 novembre 1992; accepté le 11 janvier 1993)

Résumé - Le travail présenté vise à tester la résistance de différentes provenances de pins maritimes au $\mathrm{NaCl}$ dans des conditions édaphiques proches du terrain et dans un environnement minéral le plus favorable possible. Le stress nutritionnel imposé par l'alcalinité et la pauvreté des sables littoraux de Vendée s'est avéré extrêmement péjoratif à la croissance des semis de pin en serre 11 $25 \mathrm{~g}) \mathrm{MS} / 2$ ans). Le jaunissement des aiguilles et la mortalité observés sur le sol de aPays de Monts" est bien en relation avec le caractère dépérissant du peuplement installé sur ce site et fait apparaître le caractère résistant de la provenance Tamjoute. En revanche, en ce qui concerne la production de biomasse pour le sol d'Oléron (placette saine), la différence de potentiel de croissance entre provenances ne s'exprime pas. L'effet de l'augmentation des teneurs en $\mathrm{NaCl}$ de la rhizosphère sur la croissance est peu sensible pour les pins cultivés dans ces conditions, et affecte en priorité le système racinaire. Les conditions d'alimentation minérale non limitantes (solutions nutritives coulantes) ont permis d'exprimer très nettement la toxicité au $\mathrm{NaCl}$ avec une sensibilité décroissante : "lbérique", "Landais", "Tamjoute". Compte tenu des différences de croissance entre provenances : "Landais" > "lbérique" > "Tamjoute" établies avec la solution nutritive témoin, les "Landais" conservent le meilleur taux de croissance pour toutes les doses, alors que les "lbériques" sont supplantés en terme d'accroissement par les "Tamjoute" pour les doses de $\mathrm{NaCl}$ les plus fortes.

\section{Pinus Pinaster / dépérissement / NaCl / stress salin / pH / réponse génotypique}

Summary - Maritime pine dieback on the West coast of France. Growth response to sodium chloride of 3 geographic races in various edaphic conditions. The responses of 3 geographic races of maritime pine, French ("Landais"), Iberian ("Iberique") and Moroccan ("Tamjoute") to salt stress were studied on original soils and hydroponic culture. Landais and Ibérique pine seeds were collected from pine trees from west France and the genetic origin was determined by terpene shoot analysis on trees. Tamjoute seeds were collected in the Tamjoute mountains (Morocco). Homogenous seedlings were planted in calcareous sand from forest site at the "Pays de Monts" and "Oleron" (table I) and salt stress was applied to the Oleron soil after 12 months of culture and maintained for 
$60 \mathrm{~d}$ (experiment 1$)$. In the quartz $(2-4 \mathrm{~mm})$ culture system, plants were supplied with fresh nutrient solution twice per $h$. Four stress levels were obtained with the addition of $0,50,150,250 \mathrm{mM} \mathrm{NaCl}$ to the basic solution. Total heights were measured nearly twice per month (fig 1). The 3 curves showed well-synchronized height growth. The winter dormancy was clearly marked and the $\mathrm{NaCl}$ stress was installed at the maximum and linear growth stage. The final inflexion of the curve was due to the $\mathrm{NaCl}$ toxicity. Growth in the Pays de Mont treatment was completely absent and dieback symptoms appeared at the end of the first growing season, showing that the Tamjoute pines were more resistant (table II). Dry weights of roots, stems, and needles were evaluated after the stress period for the Oléron soil (tables III, IV). Genotypic response was not significant and the salt stress slowly reduced final biomass and particulary root weight. Analyses of variance showed significant differences in final harvest weights among geographic races in the second experiment (table $V$ ). The most vigorous race was found to be the Landais with $22 \%$ gain compared with the lberique, and $36 \%$ gain compared with the Tamjoute race. Dry matter allocation was very similar between Landais and lbérique, but conversely, Tamjoute showed stronger root allocation. Toxic symptoms of $\mathrm{NaCl}$ on plant organs were not detected after 60 d culture at $250 \mathrm{mM} \mathrm{NaCl}$ concentrations but the final biomasses were reduced substantially $40 \%$ less than the control (table VI). Growth (fig 2) was calculated by substracting the final weights from the initial weights of the same plant and estimated by linear regression on the total height. The regressions were established from data of the first harvest for each geographic race (table VII). Tamjout appeared to be more resistant to salt stress than Landais on Tamjoute.

Pinus pinaster / dieback / NaCl / salt stress / pH / genotypic response

\section{INTRODUCTION}

Les observations et les études réalisées par Bonneau (1969), Boudaud (1986) et Guyon (1991) permettent de mettre en relation le dépérissement du pin maritime constaté sur la côte vendéenne, avec les caractéristiques édaphiques des dunes littorales : un $\mathrm{pH}$ élevé (supérieur à 8 ) attribuable à la fois à la présence de calcaire et à une forte teneur en sodium du sol. Cela suggère donc un dysfonctionnement métabolique lié à l'une ou l'autre cause, ou aux 2 , éventuellement exagéré par les circonstances climatiques défavorables puisque le dépérissement présente un caractère cyclique. Les symptômes du dépérissement débutent par un jaunissement de la pousse en élongation, l'avachissement des aiguilles vertes sur les branches basses, puis la généralisation du phénomène jusqu'à la défoliation (Grouhel, 1991).

Notre dispositif est destiné à tester l'hypothèse de la toxicité du chlorure de sodium sur des plants de 2 ans cultivés en conditions contrôlées, à la fois dans les conditions très drastiques des sols de la zone concernée et dans un environnement minéral très favorable en solution nutritive, qui permet de respecter des propriétés mécaniques proches d'un sol et un développement radiculaire normal, tout en contrôlant précisément l'alimentation minérale.

Cette étude porte sur 3 provenances géographiques de pin maritime "Landaise" "lbérique" et marocaine : "Tamjoute", dont on déterminera les sensibilités respectives.

\section{MATÉRIEL ET MÉTHODE}

\section{Matériel végétal}

Les graines de pin maritime "Landaises" et "lbériques" proviennent de la récolte effectuée par Hervé Magnin (1990) en forêt domaniale des Pays de Monts (Vendée) dont l'origine génétique des arbres récoltés a été déterminée par analyse des tissus corticaux des jeunes rameaux de l'année de 30 arbres, selon la méthode décrite par Baradat et Marpeau-Bézard (1988) : 
- "Landaise" : lot Magnin 76, récolte 1990 ;

- «lbérique» : lot Magnin 42, récolte 1990.

Les graines "Tamjoutes" proviennent d'une récolte effectuée au Maroc par le laboratoire d'amélioration des arbres forestiers (INRA Bordeaux) : “Tamjoute” lot INRA 86 800-86 493, récolte 1985.

\section{Conduite de la culture en serre}

Les graines ont été mises à germer le 5 avril 1991 dans de la tourbe blonde naturelle en chambre de germination et ont été repiquées le 6 mai 1991 dans leur substrat définitif, sous un tunnel plastique équipé d'un système de refroissement par ventilation forcée asservie pour une température supérieure de $30^{\circ} \mathrm{C}$.

\section{Protocole}

\section{Expérimentation 1 sur sols forestiers}

Les sols utilisés dans cette expérimentation ont été prélevés sous couvert forestier en mars 1991 par Hervé Magnin $\left(1,5 \mathrm{~m}^{3}\right)$ sur $20 \mathrm{~cm}$ épaisseur $\left(A_{1}\right)$ après suppression de $\left.A_{00}\right)$ :

- "Pays de Monts", Vendée, parcelle 42, placette DEP : peuplement de pin maritime dépérissant, absence de sous-étage ;

- "Oléron", Charente-Maritime, forêt de SaintTrojan, parcelle 43 : peuplement de pin maritime adulte, sous-étage de chêne vert.

Ces 2 sols sont des sables riches en calcaire et se caractérisent, avec une tendance plus marquée pour le sol du "Pays de Monts", par un $\mathrm{pH}$ très élevé, de fortes teneurs en $\mathrm{Ca}$ et $\mathrm{Na}$, et une pauvreté marquée en phosphore échangeable (tableau I). Les très fortes teneurs en calcium "échangeables", nettement supérieures à la capacité d'échange cationique, s'expliquent par une fraction importante de calcium soluble dans ces sols (Gelpe, communication personnelle). Les capacités d'échange cationique sont faibles dans les 2 cas.

Chaque pin est cultivé dans un pot de 4 I (4 kg MS sol "Oléron", 4,2 kg MS sol "Pays de Monts") maintenu à humidité constante $(40 \%$ capacité au champ) par irrigation automatique asservie par pesée d'un pot témoin.
Les 3 provenances de pin répétées 40 fois (120 plants par sol) ont été cultivées pendant 2 saisons de végétation.

Quatre traitements ont été appliqués au sol d' "Oléron" en 2 fois, le $1^{\text {er }}$ et le 7 mai 1992 :

- témoin (pas de stress salin) ;

- pulvérisations foliaires de $\mathrm{NaCl}$ à $20 \mathrm{~g} \cdot \mathrm{l}^{-1}$;

- arrosage du sol avec $4 \mathrm{~g} \mathrm{NaCl} /$ pot : correspond à environ $135 \mathrm{mM} / \mathrm{l} \mathrm{NaCl}$ dans la solution du sol $(500 \mathrm{ml}$ d'eau contenus dans chaque pot) ;

- arrosage du sol ave $8 \mathrm{~g} \mathrm{NaCl} /$ pot : correspond à environ $270 \mathrm{mM} / \mathrm{l} \mathrm{NaCl}$ dans la solution du sol (500 $\mathrm{ml}$ d'eau contenus dans chaque pot).

L'ensemble des plants ont été récoltés 2 mois plus tard.

\section{Expérimentation 2 en solution nutritive}

Elle est réalisée en pot de $4 \mathrm{I}$, sur support inerte (quartz grossier $2-4 \mathrm{~mm}$ ), en solution hydroponique coulante dont la composition exprimée en mol $\cdot \mathrm{m}^{-3}$ est la suivante: $\mathrm{NH}_{4} \mathrm{NO}_{3}=2$ $\mathrm{KH}_{2} \mathrm{PO}_{4}=0,5, \mathrm{CaCl}_{2}=0,25, \mathrm{MgSO}_{4}=0,25$, $\mathrm{Fe}=0,1, \mathrm{~B}=8,0.10^{-3}, \mathrm{Mn}=1,5.10^{-3}, \mathrm{Zn}=$ 1,5.10-3, $\mathrm{Cu}=0,15 \cdot 10^{-3}, \mathrm{Co}=0,0015 \cdot 10^{-3}$, $\mathrm{Mo}=0,0015 \cdot 10^{-3}$ pour un $\mathrm{pH}$ ajusté autour de 4,5 .

Les 3 provenances de pin sont répétées 80 fois (240 plants) et cultivées avec la solution nutritive de base jusqu'au 30 avril 1992, date de la récolte de 16 individus par provenance et de l'application de 4 traitements par provenance ré pétés 16 fois:

- témoin (solution nutritive) conductivité $=1,01$ $\mathrm{mS}$;

- niveau 1 (solution nutritive $+50 \mathrm{mM} / \mathrm{l}$ de $\mathrm{NaCl}$ ) conductivité $=6,29 \mathrm{mS}$;

- niveau 2 (solution nutritive $+150 \mathrm{mM} / \mathrm{l}$ de $\mathrm{NaCl}$ ) conductivité $=14,3 \mathrm{mS}$;

- niveau 3 (solution nutritive $+250 \mathrm{mM} / \mathrm{l}$ de $\mathrm{NaCl}$ ) conductivité $=24,8 \mathrm{mM}$

La récolte définitive a eu lieu le 9 juillet 1992.

Pour l'ensemble des expérimentations, la hauteur des plants a été mesurée environ toutes les 2 semaines et les biomasses sèches $\left(80^{\circ} \mathrm{C}\right)$ déterminées à la récolte des pins pour les racines, les tiges et les aiguilles.

Dans l'expérimentation 2, les biomasses des plants avant l'installation du stress (30 avril 
Tableau I. Caractéristiques physico-chimiques des sols utilisés dans l'expérimentation 1.

\begin{tabular}{|c|c|c|}
\hline & "Pays de Monts" & "Oléron" \\
\hline \multicolumn{3}{|l|}{ Granulométrie $(\mathrm{g} / \mathrm{kg})$} \\
\hline Argile & 22 & 21 \\
\hline Limon fin & 1 & 0 \\
\hline Limon grossier & 0 & 0 \\
\hline Sable fin & 339 & 655 \\
\hline Sable grossier & 638 & 324 \\
\hline C organique (méthode Anne) & 8,1 & 7,3 \\
\hline Matières organiques & 13,9 & 12,6 \\
\hline N Kjeldahl $(g / k g)$ & 0,48 & 0,32 \\
\hline $\mathrm{C} / \mathrm{N}$ & 16,88 & 22,81 \\
\hline pH eau & 8,4 & 7,3 \\
\hline $\mathrm{P}_{2} \mathrm{O}_{5}$ Duchaufour $(\mathrm{g} / \mathrm{kg})$ & 0,01 & 0,07 \\
\hline Calcaire total $(\mathrm{g} / \mathrm{kg})$ & 179 & 40 \\
\hline - Calcaire actif $(g / k g)$ & 15 & 7 \\
\hline CEC Metson (Meq/kg) & 35 & 28 \\
\hline Ca échangeable (Meq/kg) & 347 & 137 \\
\hline $\mathrm{Na}$ échangeable (Meq/kg) & 2,19 & 0,85 \\
\hline Mg échangeable (Meq/kg) & 11,3 & 6,7 \\
\hline K échangeable (Meq/kg) & 0,32 & 0,76 \\
\hline Mn échangeable (mg/kg) & 13,3 & 5,3 \\
\hline
\end{tabular}

* Grounel (1991).

1992) sont estimées à partir de la hauteur mesurée à cette date par une régression linéaire entre hauteur et biomasse établie pour chaque tissu et pour chaque provenance avec les 48 plants sacrifiés à cet effet. L'accroissement des plants en biomasse pendant la période de 70 j de stress est calculée pour chaque plant par la différence entre la biomasse estimée avant le traitement et la biomasse mesurée après.

\section{RÉSULTATS}

\section{Expérimentation 1 sur sols forestiers}

L'élevage des plants sur le sol forestier du «Pays de Monts» a constitué un stress minéral extrêmement important, provoquant 
des symptômes de chlorose très marqués, se traduisant par une croissance en hauteur insignifiante (fig 1), un jaunissement du feuillage et une mortalité importante en deuxième saison de végétation (tableau II). Le taux d'individus sains de $65 \%$ de la provenance «Tamjoute» est très nettement supérieur à celui des 2 autres provenances (25 et $10 \%$ ) (test du $\chi^{2}, P=0,004$ ). Les biomasses totales des individus sains étaient de 3,1 g MS pour les “Tamjoutes" contre 1,1 g MS pour les "Landais". Les effectifs restants étant trop réduits nous n'avons pu tester l'interaction de ce sol avec la toxicité en chlorure de sodium.

La culture sur le sol d' "Oléron" a donné de meilleurs résultats, avec une hauteur de $12 \mathrm{~cm}$ en fin de première saison de végétation et un bon redémarrage de la croissance la deuxième année (fig 1). Le stress salin a été établi en pleine croissance à partir du $1^{\text {er }}$ mai. La mesure des biomasses sèches au moment de la récolte ne fait pas apparaître de différences significatives entre les croissances totales
Tableau II. Pourcentage d'individus sains, morts ou présentant un jaunissement des aiguilles pour l'expérimentation 1 sur sol de Pays de Monts, en l'absence de traitement au chlorure de sodium $\left(x^{2}=15,3, d f=4, P=0,004\right)$.

\begin{tabular}{llll}
\hline Provenance & Sain & Jaune & Mort \\
& & & \\
\hline "Landais" & 25 & 20 & 55 \\
"bérique" & 10 & 35 & 55 \\
"Tamjout" & 65 & 10 & 25 \\
\hline
\end{tabular}

des différentes provenances (environ $25 \mathrm{~g}$ MS/ind), mais montre la nette tendance des "Tamjoutes" à favoriser le système racinaire aux dépens des parties aériennes (tableau III). Le tableau IV nous indique que le traitement au $\mathrm{NaCl}$ pulvérisé sur le feuillage n'a pas eu d'effet sur la croissance, tandis que la dose $2(8 \mathrm{~g} \mathrm{NaCl} /$ pot) a faiblement réduit la biomasse finale $(10 \%)$, en affectant principalement le système racinaire.

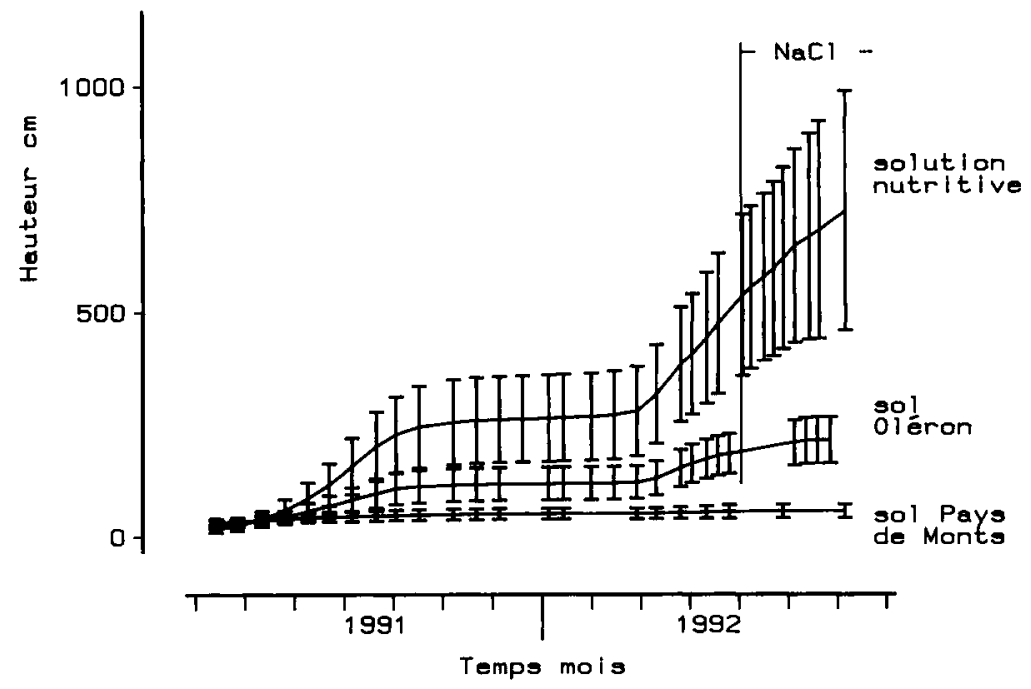

Fig 1. Courbe de croissance en hauteur des pins, toutes provenances et tous traitements confondus, pour l'expérimentation 1 (sol Oléron et Pays de Monts) et l'expérimentation 2 en solution nutritive coulante. L'application du stress salin est indiquée par une barre verticale. Les intervalles figurés représentent 4 écarts types. 
Tableau III. Biomasse en g MS/individu des pins de l'expérimentation 1 sur sol d'Oléron en fonction de la provenance tous traitements confondus.

\begin{tabular}{lcccc}
\hline Provenance & Racines & Tiges & Aiguilles & Total \\
\hline & & & & \\
\hline & & & & \\
"Landais" & $7,2 \mathrm{~b}$ & $3,7 \mathrm{a}$ & $13,9 \mathrm{a}$ & $25,9 \mathrm{a}$ \\
"lbérique" & $7,4 \mathrm{~b}$ & $3,7 \mathrm{a}$ & $13,7 \mathrm{ab}$ & $24,7 \mathrm{a}$ \\
"Tamjout" & $10,0 \mathrm{a}$ & $3,2 \mathrm{~b}$ & $12,7 \mathrm{~b}$ & $25,9 \mathrm{a}$ \\
\hline
\end{tabular}

'Dans une même colonne, 2 valeurs suivies de la méme lettre ne diffèrent pas significativement au seuil de $10 \%$ (test de Duncan).

Tableau IV. Biomasse en g MS/individu des pins de l'expérimentation 1 sur sol d'Oléron en fonction du traitement $\mathrm{NaCl}$, toutes provenances confondues.

\begin{tabular}{lllll}
\hline Traitement & Racines & Tiges & Aiguilles & Total \\
\hline Témoin & $9,15^{4}$ & $3,8 \mathrm{a}$ & $13,9 \mathrm{a}$ & $26,8 \mathrm{a}$ \\
Pulvérisé & $9,2 \mathrm{a}$ & $3,4 \mathrm{a}$ & $13,3 \mathrm{a}$ & $25,9 \mathrm{ab}$ \\
Dose 1 & $7,7 \mathrm{~b}$ & $3,5 \mathrm{a}$ & $13,7 \mathrm{a}$ & $25,0 \mathrm{ab}$ \\
Dose 2 & $8,0 \mathrm{~b}$ & $3,4 \mathrm{a}$ & $12,8 \mathrm{a}$ & $24,2 \mathrm{~b}$ \\
& & & & \\
\hline
\end{tabular}

'Dans une même colonne, 2 valeurs suivies de la même lettre ne diff!èrent pas significativement au seuil de $10 \%$ (test de Duncan).

\section{Expérimentation 2 en solution nutritive}

La croissance en hauteur moyenne pour ce type de culture est très largement supérieure à celle obtenue sur sol de par une alimentation minérale non limitante (fig 1). L'augmentation des intervalles de confiance sur la courbe de croissance (fig 1) à partir de l'établissement du stress, traduit la toxicité du chlorure de sodium.

Les biomasses finales obtenues par cette culture en solution nutritive non carencée permettent de discriminer très nettement les 3 provenances (tableau V). Si le classement "Landais" > «lbérique" >
"Tamjoute" établi sur la biomasse totale est conservé pour tous les tissus considérés, le «Tamjoute» se distingue par une répartition différente de la matière sèche : $18 \%$ de racines, $26 \%$ de tiges, $55 \%$ d'aiguilles, contre $14 \%$ de racines, $35 \%$ de tiges, $51 \%$ d'aiguilles pour les "Landais" et "lbériques". L'effet des traitements $\mathrm{NaCl}$ est spectaculaire sur la production de biomasse à partir de la dose $150 \mathrm{mM} / \mathrm{l}$ (tableau VI). Pour la dose $250 \mathrm{mM} / \mathrm{l}$, les pertes en biomasse par rapport au témoin sont respectivement de $38 \%, 42 \%$ et $40 \%$ pour les racines, les tiges et les aiguilles, toutes les provenances confondues. 
Tableau V. Biomasse en g MS/individu des pins de l'expérimentation 2 en fonction de la provenance après $70 \mathrm{j}$ de stress salin, tous traitements confondus.

\begin{tabular}{lllll}
\hline Provenance & Racines & Tiges & Aiguilles & Total \\
\hline "Landais" & $15,4^{\circ}$ & $38,1 \mathrm{a}$ & $55,9 \mathrm{a}$ & $109,5 \mathrm{a}$ \\
"Ibérique" & $12,6 \mathrm{~b}$ & $30,9 \mathrm{~b}$ & $46,1 \mathrm{~b}$ & $89,6 \mathrm{~b}$ \\
"Tamjout" & $12,9 \mathrm{~b}$ & $18,1 \mathrm{c}$ & $38,5 \mathrm{c}$ & $69,5 \mathrm{c}$ \\
\end{tabular}

'Dans une même colonne, 2 valeurs suivies de la même lettre ne diffèrent pas significativement au seuil de $10 \%$ (test de Duncan).

Tableau VI. Biomasse en g MS/individu des pins de l'expérimentation 2 en fonction du traitement $\mathrm{NaCl}$ après 70 j de stress salin, toutes provenances confondues.

\begin{tabular}{ccccc}
\hline Traitement $(m M / l)$ & Racines & Tiges & Aiguilles & Total \\
\hline 0 & $16,8 \mathrm{a}$ & $37,5 \mathrm{a}$ & $57,6 \mathrm{a}$ & $112,0 \mathrm{a}$ \\
50 & $16,1 \mathrm{a}$ & $32,2 \mathrm{~b}$ & $51,9 \mathrm{a}$ & $100,0 \mathrm{a}$ \\
150 & $11,2 \mathrm{~b}$ & $24,4 \mathrm{c}$ & $43,2 \mathrm{~b}$ & $78,8 \mathrm{~b}$ \\
250 & $10,4 \mathrm{~b}$ & $21,8 \mathrm{c}$ & $34,5 \mathrm{c}$ & $66,7 \mathrm{~b}$ \\
\hline
\end{tabular}

'Dans une même colonne, 2 valeurs suivies de la même lettre ne diffèrent pas significativement au seuil de $10 \%$ (test de Duncan).

Des régressions linéaires entre hauteur et biomasse (racine, tige, feuille) des plants ont été établies par échantillonnage de 48 plants avec l'application du stress pour chacune des provenances (tableau VII). Ces résultats nous ont permis d'estimer les biomasses au début du traitement et de calculer des accroissements pondéraux pour chaque individu (fig 2). L'effet du traitement $\mathrm{NaCl}$ sur l'accroissement des différents tissus, testé par une analyse de variance, s'avère hautement significatif pour chaque provenance (tableau VIII). La croissance racinaire est pratiquement stoppée à partir de la dose $150 \mathrm{mM} / \mathrm{l}$, bien que les «Tamjoutes" résistent encore avec une production de $2 \mathrm{~g}$ MS. Les tiges et les ai- guilles sont sensibles à la toxicité saline, mais conservent une croissance non négligeable aux doses les plus fortes. La sensibilité au stress, estimée par le taux de réduction de croissance à la dose de $250 \mathrm{mM} / \mathrm{l}$, est de $84,6 \%$ pour les "lbériques", 70,4\% pour les "Landais" et $66,4 \%$ pour les “Tamjoutes". Malgré leur plus grande sensibilité au sel, les "Landais" conservent une croissance supérieure aux «Tamjoute" par leur potentialité de croissance supérieure ; par contre pour la dose critique de $150 \mathrm{mM} / / \mathrm{NaCl}$, les "Tamjoutes" assurent une croissance plus forte que les "lbériques", en dépit de leur potentiel de croissance, faible en conditions non stressantes. 
Tableau VII. Paramètres des régressions linéaires entre hauteurs des plants et biomasses avant l'établissement du stress.

\begin{tabular}{lllll}
\hline Provenance & $R^{2}$ & Pente & $\operatorname{Pr}>T$ & $E T^{*}$ \\
\hline $\begin{array}{l}\text { Racines } \\
\text { "Landais" }\end{array}$ & 0,46 & & & \\
"lbérique" & 0,71 & 0,021 & 0,0038 & 0,0062 \\
"Tamjout" & 0,48 & 0,016 & 0,0001 & 0,0027 \\
& & 0,022 & 0,0042 & 0,0063 \\
Tiges & 0,59 & & & \\
"Landais" & 0,90 & 0,051 & 0,0005 & 0,0116 \\
"lbérique" & 0,78 & 0,037 & 0,0001 & 0,0034 \\
"Tamjout" & & 0,044 & 0,0001 & 0,0065 \\
& 0,43 & & & \\
Aiguilles & Landais" & 0,065 & 0,0060 & 0,0201 \\
"lbérique" & 0,87 & 0,049 & 0,0001 & 0,0052 \\
"Tamjout" & 0,48 & 0,060 & 0,0041 & 0,0174 \\
\hline
\end{tabular}

'ET : Écart type des valeurs estimées.

Tableau VIII. Signification statistique de l'effet des doses de $\mathrm{NaCl}$ sur les accroissements en biomasses des 3 provenances présentées sur la fig 2 : analyse de variance.

\begin{tabular}{lccc}
\hline Provenance & $d l$ & $F$ & $\operatorname{Pr}>F$ \\
\hline $\begin{array}{l}\text { Racines } \\
\text { "Landais" }\end{array}$ & 64 & 9,39 & \\
"lbérique" & 62 & 19,9 & 0,0001 \\
"Tamjout" & 64 & 12,37 & 0,0001 \\
& & & 0,0001 \\
Tiges & 64 & 6,24 & \\
"Landais" & 62 & 18,12 & 0,0009 \\
"Ibérique" & 4,66 & 0,0001 \\
"Tamjout" & 64 & & 0,0054 \\
Aiguilles & & 6,28 & \\
"Landais" & 64 & 13,97 & 0,0009 \\
"lbérique" & 7,38 & 0,0001 \\
"Tamjout" & 62 & & 0,0003 \\
\hline
\end{tabular}




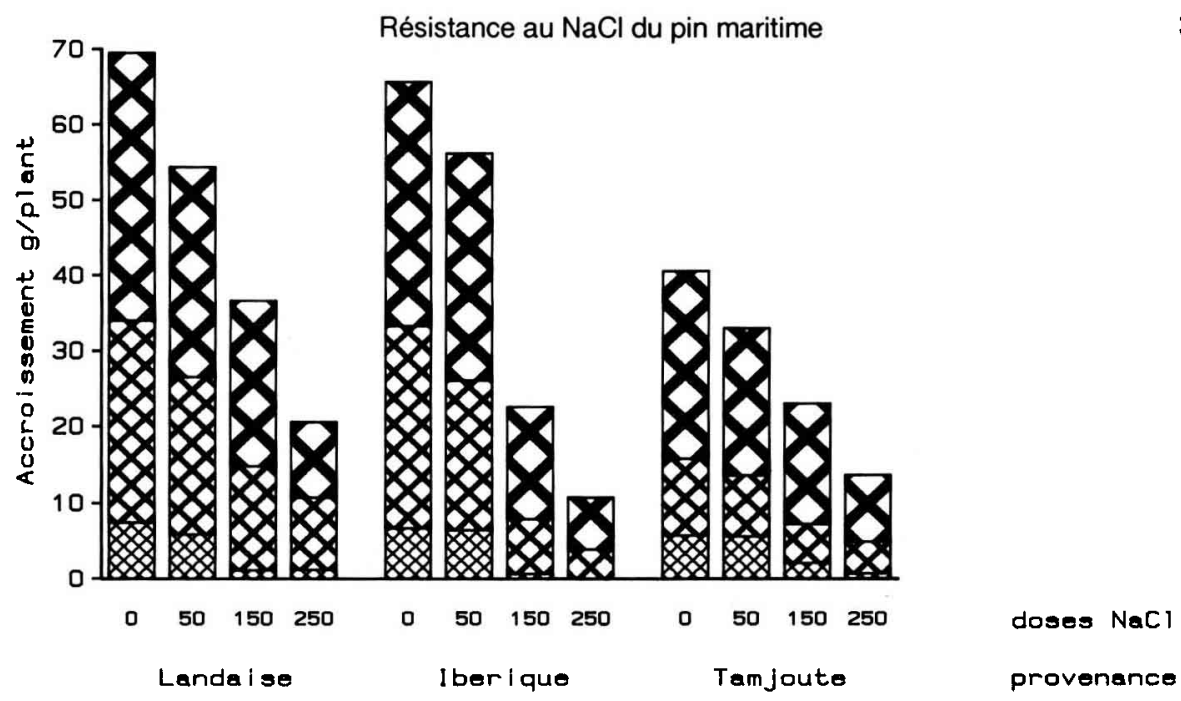

Fig 2. Accroissement des plants en matière sèche pendant les $70 \mathrm{j}$ de traitement au chlorure de sodium en fonction de la dose de $\mathrm{NaCl}$ (exprimée en $\mathrm{mM} / \mathrm{l}$ ) et de la provenance des pins pour les racines, les tiges et les aiguilles. Tissus : racine ( tige (

\section{DISCUSSION ET CONCLUSIONS}

\section{Alcalinité du sol}

Le stress nutritionnel imposé par l'alcalinité et la pauvreté des sables littoraux de Vendée s'est avéré extrêmement péjoratif à la croissance des semis de pin en serre (1$25 \mathrm{~g} \mathrm{MS} / 2$ ans). Le jaunissement des aiguilles et la mortalité observés sur le sol de "Pays de Monts" est bien en relation avec le caractère dépérissant du peuplement installé sur ce site, par comparaison avec le sol d' "Oléron" (peuplement sain). II y a, dans ce cas, adéquation entre les observations de terrain et l'expérimentation en serre, bien que les effets toxiques ou défavorables du sol soient exacerbés en en serre, principalement par des effets de température du sol ; la régénération naturelle est en effet satisfaisante sur la parcelle de "Pays de Monts" (Guyon, communication personnelle). En ce qui concerne le comportement des 3 prove- nances face à ce stress, le caractère résistant de la provenance "Tamjoute» apparaît nettement sur le taux de survie pour le sol le plus défavorable. Cette caractéristique est à mettre en relation avec les stations marocaines d'origine caractérisées par des sols calcaires. En revanche, en ce qui concerne la production de biomasse en condition limitante, la différence de potentiel de croissance entre provenances ne s'exprime pas (sol “Oléron»).

\section{Toxicité au chlorure de sodium}

L'effet de l'augmentation des teneurs en $\mathrm{NaCl}$ de la rhizosphère sur la croissance est peu sensible pour les pins cultivés en condition de stress nutritionnel et affecte en priorité le système racinaire. L'application foliaire massive n'a pas eu de conséquence sur la croissance dans les conditions précédentes, ce qui ne présume pas des effets à long terme par dégradation des cires épicuticulaires, mais exclut une 
absorption importante et rapide par cette voie en l'absence de tensioactifs (Gellini et al, 1987). Des conditions d'alimentation minérale non limitantes ont permis d'exprimer très nettement la toxicité au $\mathrm{NaCl}$, avec une sensibilité décroissante : "lbérique», "Landais", “Tamjoute». Ces résultats sont en accord avec Heller (1989), qui classe le pin maritime dans les plantes sensibles au chlorure de sodium, qui accusent une baisse de rendement de $20 \%$ pour des doses de $30-50 \mathrm{mM} / \mathrm{l}$. Parmi les essences forestières, il présente une résistance non négligeable, supérieure aux expèces les plus sensibles comme Tilia, Alnus incana ou Fagus sylvatica, qui ne tolèrent pas des doses de 34 $\mathrm{mM} / \mathrm{l} \mathrm{NaCl}(2 \mathrm{~g} / \mathrm{l})$, alors que les espèces les plus résistantes comme Populus alba ou $P$ canescens supportent des doses de $154 \mathrm{mM} / \mathrm{l} \mathrm{NaCl}$ d'après Glasau (1966) cité par Bonneau (1980). Par ailleurs, Pezeshki (1991) décrit une toxicité sur Pinus taeda pour des doses de $50 \mathrm{mM} / \mathrm{l}$, mais la variabilité génétique de la réponse au stress salin sur des espèces de pin n'a pas à notre connaissance fait l'objet d'étude jusqu'alors. Compte tenu des différences de croissance entre provenances: "Landais" > «lbérique" > “Tamjoute" établies avec la solution nutritive témoin, les "Landais" conservent leur meilleur taux de croissance pour toutes les doses, alors que les «bériques" sont supplantés en terme d'accroissement par les “Tamjoutes", pour les doses de $\mathrm{NaCl}$ les plus fortes.

II est remarquable de noter que les résultats obtenus sur des plants de 2 ans en solution nutritive exacerbent les effets "provenance» et «toxicité" masqués par une culture sur le sol forestier d'origine trop stressant, et sont en accord avec les résultats de terrain sur arbres adultes en ce qui concerne la différence "Landais»/ "lbérique». En effet, il a été établi par Guyon (1991) que les ralentissements de croissance dans les parcelles dépérissantes sont plus accusés chez les populations ibériques que chez les populations acquitaines. Ce constat étaie la validité d'une étude écophysiologique sur ce type de matériel végétal (appareil foliaire constitué d'aiguilles adultes : "pseudophylles»), et dans les conditions minérales favorables à l'étude de l'expression de la toxicité, afin de décrire les mécanismes impliqués et la variabilité intraspécifique dans la réponse au stress salin.

\section{REMERCIEMENTS}

Cette étude a été réalisée en collaboration avec D Loustau, grâce au soutien financier de l'Office national des forêts, région Pays de Loire, dans le projet "Résistance au sel", coordonné par JP Guyon. Nous remercions vivement A Pauillac qui a pris en charge tout le dispositil de culture, ainsi que S Crepeau, JL Grange, M Guedon et M Sartore pour leur collaboration technique.

\section{RÉFÉRENCES}

Baradat P, Marpeau-Bézard A (1988) Le pin maritime. Biologie et génétique des Terpènes pour la connaissance et l'amélioration de l'espèce. Thèse de doctorat d'État, université de Bordeaux 1, $645 \mathrm{p}$

Boudaud JR (1986) Étude de la forêt des Pays de Monts". Mémoire de maîtrise de l'université de Nantes, $75 p$

Bonneau M (1969) Dépérissement des pins maritimes des forêts de la Barre de Monts et de Saint-Jean-de-Monts. Commentaire des analyses de sols et des analyses foliaires. INRA Rapport, $15 \mathrm{p}$

Bonneau M (1980) Appréciation de la fertilité du sol. Éléments autres que l'azote : $P, K, \mathrm{Ca}$, $\mathrm{Mg}$, oligo-éléments. Phénomène de toxicité. Cours de pédologie forestière. ENGREF, 2-5 $28 p$

Gellini R, Pantani F, Grossoni P, Bussotti $F$ (1987) L'influence de la pollution marine sur la végétation côtière italienne. Bull Ecol 18, 213-219 
Grouhel A (1991) Dépérissement du pin maritime sur le littoral Atlantique (Vendée et Charente-Maritime). Aspects méthodologiques, géomorphologie et symptomatologie. Mémoire DAA, ENSA Rennes, $104 \mathrm{p}$

Guyon JP (1991) Dépérissement du pin maritime (Pinus pinaster Aït) en Vendée. Les causes écologiques. Ann Sci For 48, 333346
Heller R (1989) Physiologie végétale.1.Nutrition. Masson, Paris, $273 \mathrm{p}$

Magnin H (1990) Dépérissement du pin maritime sur le littoral vendéen. État sanitaire des peuplements et écologie du dépérissement. Mémoire de $3^{\mathrm{e}}$ année ENITEF, $76 \mathrm{p}$

Pezeshki SR (1992) Response of Pinus taeda L to soil flooding and salinity. Ann Sci For 49, 134-149 